\title{
The Influence of Optimistic Expectations and Negative Life Events on Somatic Symptoms among Adolescents: A One-Year Prospective Study
}

\author{
Terje Arnfinn Murberg \\ University of Stavanger, Stavanger, Norway \\ Email: terje.a.murberg@uis.no
}

Received October $20^{\text {th }}, 2011$; revised December $9^{\text {th }}, 2011$; accepted January $12^{\text {th }}, 2012$

\begin{abstract}
This study prospectively examined the main effect of optimism on subsequent somatic symptomatology as well as optimism as moderating factors in the link between negative life events and somatic symptoms in a sample of 198 (111 females, 87 males) students in a Norwegian senior high school. Results from the longitudinal multivariate analyses, indicated that the scores for optimism and negative life events were significantly associated with scores of somatic symptoms at time-point two $\left(\mathrm{T}_{2}\right)$. Moreover, a significant Optimism $\times$ Negative life events interaction was found in predicting somatic symptoms. Implications of these findings are discussed.
\end{abstract}

Keywords: Optimism; Negative Life Events; Somatic Symptoms; Senior High School Students

\section{Introduction}

Physical symptoms of unknown pathology have haunted medical and mental health practitioners for centuries. Somatisation problems often start in adolescence and continue into adult life. It is estimated that $10 \%$ to $15 \%$ of all youngsters report somatic complaints (Campo \& Fritsch, 1994, Ryan-Wenger, 1994). In addition to their high prevalence, finding indicate that somatic symptoms are associated with high levels of co-morbid psychological symptoms, like anxiety and depression (Haug, Mykletun, \& Dahl, 2004), as well as high levels of functional impairment (Mulvaney et al., 2006; Aromaa et al., 2000). Given that these symptoms are linked to poor adaption during adolescence, such as decreased work and school productivity (Aro, 1987) and increased used of the health care system, understanding early risk and protective factors for somatic symptoms is crucially important (Di Lorenzo et al., 2005).

Different theoretical approaches have dominated the research in understanding somatic symptomatology. Empirical support has been found for the idea that life events perceived as negative or stressful for individuals have the potential to produce somatic symptoms. Adolescence is a challenging period of life that includes experience of stressful life events that may be critical for the individual's developmental and psychological adjustment. Different acute or chronic stressful life events impose demands that some people are unable to cope with. Such negative experiences are considered to put individuals at risk for psychological and physical problems. A number of studies have reported a significant association between stressful life events and somatic symptoms among adolescents (Boey \& Goh, 2001; Walker, Garber, \& Greene, 1994). Longitudinal studies exist indicating that negative life events are associated with psychological and physical health problems in adolescents (see Waaktaar et al.; Ystgaard, Tams, \& Dalgaard, 1999). However, it is not the experience of stressful life events per se that is necessarily harmful, but often the individual's inability to cope adequately with various stressful events that have a negative impact on the adolescent's health and well-being. Unpredictable or disruptive environments may undermine an adolescent's sense of control and mastery, leading to a sense of helplessness and/or hopelessness that acts as a precursor to somatic symptomatology. It is therefore predicted that the experience of negative life events would increase the risk of somatic symptoms in this sample of mid-adolescents.

While stress is a part of the adolescent's life, the same negative life event may not be perceived as equally stressful by different individuals. The experience of stress is influenced by the individual's perception and interpretations (Lazarus \& Folkman, 1984). Some researchers have considered the role of cognitive-affective factors, which may facilitate coping with the demands imposed by stressful life events, and consequently ameliorate the potentially negative influences of stressful life events and thus be protective (Skodol, 1998). Dispositional optimism, which is conceptualized as the generalized tendency to expect positive outcomes in the future, even under stressful situations (Scheier, Carver, \& Bridges, 2000), is one internal personality factor which may influence the way a person appraises negative life events, his or her reactions to the events, and thus the outcomes (Mäkikangas \& Kinnunen, 2003). Thus, dispositional optimism should be considered as a protective factor for somatic health problems (Mosing et al., 2010; Chang \& Sanna, 2003). The individual's optimistic view may provide ongoing feelings of security, positive affect, and self-efficacy. Consequently, optimists may expect to cope effectively with stressful life events, whereas pessimists are those who do not expect to cope successfully (Scheier \& Carver, 1985, 1992). Optimists are reported to use more active, problem-focused coping strategies in managing stressful events, whereas less optimistic individuals are likely to adopt avoidance strategies in similar situations (Scheier \& Carver, 1985). The abilities to cope actively may reduce the adverse physio- 
logical effects of negative life events (Taylor et al., 2000). In a study of students entering college, Aspinwall and Taylor (1992), found that optimistic students were likely to use active coping, which in turn predicted better adjustment to college. Thus, it is predicted that somatic symptoms will be less pronounced among adolescents scoring high on optimism, compared with their more pessimistic counterparts.

However, studies of optimism have been found to moderate the association between stressful life events and psychological and physical adjustment outcomes among adolescents (Lai, 2009; Chang \& Sanna, 2003; Chang, 1998b). The buffer or moderator effects of optimism imply that adolescents who report high levels of stress, but are likely to have positive emotional reactions and expectations, may be more likely to be protected from the negative effects of life stressors than those who are more pessimistic. However, relatively little research has focused on the role that optimism plays in the mitigation of stressful events and the reduction of psychopathology, and most studies that have examined the effects of optimism on adolescent's health have been cross-sectional. Consequently, developmental and causal conclusions have been somewhat tenuous. Therefore, more prospective studies are needed to investigate the potential buffering effects of optimism on somatic symptoms among late adolescents. In the present study, we examined the stress buffering effects of optimism in a sample of adolescents in senior high school who experienced negative life events. The specific hypothesis is that a Negative life events $x$ Optimism interaction reduces the effects of negative life events on somatic symptoms among adolescents scoring high on optimism.

In summing up, the purpose of the present study was to 1) examine the relationship between optimism, negative life events, and somatic symptoms; and 2) determine whether optimism moderates the link between negative life events and somatic symptomatology.

\section{Control Variables}

It is well documented across disciplines that children with somatic symptoms display elevated levels of psychological symptoms, such as depression or anxiety (Garber, Walker, \& Zeman, 1991; Egger, Angold, \& Costello, 1998), and that the frequency of somatic symptoms tends to increase with the severity of anxiety and depressive symptoms (Dhossche et al., 2001). Depressive symptoms were therefore included as a covariate in the present study.

\section{Methods}

\section{Participants}

The sample consisted of senior high school students who were 16 - 18 years old at baseline. The sample was recruited from one senior high school in a city in South West Norway. Participation in the study was voluntary and required both written parent and student consent. Consent was obtained from $87 \%$ of the eligible students in the school. Data were collected at two time points. The second assessment $\left(\mathrm{T}_{2}\right)$ occurred 1 year after the first $\left(T_{1}\right)$ assessment in the same winter month of each year. The present analyses were based on those 198 students who returned valid questionnaires at both waves, which constitutes $50 \%$ of the original sample. Most of the attrition from the original sample was a result of many students at $T_{1}$ leaving school at $\mathrm{T}_{2}$ to study at other senior high schools in the county. Attrition analyses showed that there were no significant differences between those who completed the study and those who did not complete the study on any of the study variables at baseline.

Respondents were asked to complete a questionnaire during an ordinary 45-minute classroom period with a teacher present. Students did not enter their names on the questionnaires and were assured of anonymity. Each student's school identification number was used to identify and track the questionnaire that she or he completed. To reduce the risk of students influencing each other's responses, the questionnaires were administered, as far as possible, at the same time for each class in every school.

\section{Measures}

Dispositional optimism. Optimism was assessed by the revised life orientation scale (LOT-R; Scheier, Carver, \& Bridges, 1994). This scale assesses trait-like optimism and pessimism via general dispositional outcome expectancies of the respondent. It consists of 10 statements (3 positively worded, 3 negatively worded and 4 filler items) and requires participants to indicate how strongly they agree with each statement using a five-point Likert-type scale ranging from 0 (strongly disagree) to 4 (strongly agree). Examples of items include: 1) In uncertain times, I usually expect the best; 2) If something can go wrong for me, it will; and 3) I'm always optimistic about my future. Negatively worded items are reverse scored and all items are summed to create an overall score; filler items are not included in the total score. Higher scores indicate increased optimism. Chronbach's alpha for this scale on dispositional optimism in the present study was 0.82 .

Negative life events. Negative life events were assessed by a checklist constructed on the basis of an established life events list for adolescents (Swearingen \& Cohen, 1985). The scale has been used several times among senior high school Norwegian adolescents (Ystgaard, 1997; Ystgaard, Tams, \& Dalgard, 1999). This instrument included 10 items illustrative of life events. Items that were covered, included parents divorcing or remarrying, parents becoming unemployed or disabled, self becoming ill or injured serious illness among close relatives or friends, unhappy love affair and self being harassed. Responses to items were given in two sections. In the first section, students were asked to indicate if they had experienced such an event on a dichotomous scale (Yes/No). In the second section, students were asked to appraise the strain they experienced due to the events using a five steps scoring format ranging from "little" to "very much". Chronbach's alpha for the scale on negative life events in the present study was 0.76 .

Somatic symptom. Somatic symptoms were assessed on the basis of six items from the Ursin Health Inventory (UHI: Ursin, Endresen, \& Ursin, 1988) and five items from the Hopkins Symptoms Checklist (HSCL: Derogatis et al., 1974). These items all had a four-step scoring format with the following response categories: "no complaints”, "mild complaints", "moderate complaints" and "severe complaints". The six UHI-items of psychosomatic symptoms were combined with those from the HSCL to compute index-scores for different psychosomatic symptoms. The index on exposure to psychosomatic symptoms had a Cronbach's alpha of 0.79 in the present study. 


\section{T. A. MURBERG}

Depressive symptoms. Depressive symptoms were assessed on the basis of seven items that covered depressive complaints ("feeling low in energy", "slowed down"; "crying easily", "feeling blue"; "feeling no interest in things"; "feeling everything is an effort"; "poor appetite", and "difficulty falling asleep or staying asleep”) from the 25-item version of the Hopkins Symptoms Checklist (Derogatis et al., 1974; Winokur et al., 1984). Exploratory as well as confirmatory factor analyses indicate that these items assess a uniform construct. Exploratory factor analyses, implementing principal axis factoring, oblique rotation and eigenvalue set to 1 , yielded a one-factor solution for the items that were implemented to assess depressive symptoms. This factor structure was tested by confirmatory factor analysis implementing maximum likelihood estimation. The analysis indicated a close fit for the one-factor solution $[$ RMSEA $=0.038 ; 90 \% \mathrm{CI}=(0.000,0.063)]$. The scale of depressive symptoms had a Cronbach's alpha of 0.82 . These items all had four-step scoring formats with the following response categories: "no complaints", "mild complaints", "moderate complaints" and "severe complaints".

\section{Procedures}

The selected statistical tools were descriptive analyses, product-moment-correlations, reliability testing (Cronbachs alpha), exploratory factor analysis, confirmatory factor analysis, and multiple regression analysis. Statistical analyses were conducted using SPSS-PC package, version 17 (Norusis, 2008) and AMOS (Arbuckle \& Wothke, 1999).

\section{Results}

Table 1 presents the Pearson product-moment correlations between study variables at $T_{1}$. As can be seen from the table, scores on the LOT-R were significantly, negatively correlated with negative life events, somatic symptoms, and depressive symptoms. As expected, a relatively strong positive association between negative life events and somatic symptoms appeared. For the covariate depressive symptoms, a significant, positive association with somatic symptoms and negative life events were detected.

A multiple regression analysis with two sections was conducted in order to explore the prospective effect of optimism and negative life events at $T_{1}$ on $T_{2}$ levels of somatic symptoms, controlling for initial level of somatic symptoms and depressive symptoms. In the first section, optimism and negative life events were entered simultaneously to test the main effects of each of these variables, controlling for $\mathrm{T}_{1}$ depressive symptoms. The $\mathbf{R}^{2}$ s, standardized $\beta$, and $p$ values are presented in Table 2 .

Table 1.

Pearson's intercorrelations between different study variables at $\mathrm{T}_{1}(\mathrm{~N}=$ 198).

\begin{tabular}{|c|c|c|c|c|}
\hline Variables & 1 & 2 & 3 & 4 \\
\hline 1. Optimism & - & & & \\
\hline 2. Negative life events & $-0.29^{* * *}$ & - & & \\
\hline 3. Somatic symptoms & $-0.19^{* *}$ & $0.43^{* * *}$ & - & \\
\hline 4. Depressive symptoms & $-0.32^{* * *}$ & $0.42^{* * *}$ & $0.63^{* * *}$ & - \\
\hline
\end{tabular}

Table 2.

Result from multivariate regression analyses for the prospective effects of optimism, negative life events, depressive symptoms at $T_{1}$ on somatic symptoms at $T_{2}$ (controlling for initial somatic symptoms), as well as interaction effects of optimism and negative life events on somatic symptoms at $\mathrm{T}_{2}(\mathrm{~N}=198)$.

\begin{tabular}{lcc}
\hline Variables & Standardized $\beta$ & $p$ \\
\hline Section 1 & -0.150 & 0.014 \\
Optimism & 0.371 & 0.000 \\
Negative life events & 0.362 & 0.000 \\
Somatic symptoms & 0.044 & n.s \\
Depressive symptoms & 0.427 & 0.000 \\
$\mathrm{R}^{2}$ & & \\
Section 2 & -0.033 & 0.000 \\
Optimism $\times$ Negative life events & 0.460 & 0.000 \\
Overall $\mathrm{R}^{2}$ & & \\
\hline
\end{tabular}

Results from this prospective analysis indicated a significant, negative effect of optimism on subsequent somatic symptoms, whereas for negative life events and initial level of somatic symptoms, a significant, positive effect on somatic symptoms at $T_{2}$ was found. In the second section, as a test of the stressbuffering effects, significant negative interactions with negative life events were found for optimism. The increment in $\mathrm{R}^{2}$ was $3.3 \%$. Overall, the entire set of predictor variables accounted for $46.0 \%$ of the variance in $\mathrm{T}_{2}$ somatic symptoms.

\section{Discussion}

Relatively few prospective studies have been designed to assess the effects of optimism on subsequent somatic symptoms among students in senior high school exposed to stressful life events. Thus, the first objective of this investigation was to examine the relationships among optimism and somatic symptomatology in a sample of senior high school students. Results from the multiple regression analyses showed that a high score on optimism was significantly and negatively related to somatic symptoms obtained one year later. This association was evident even when controlling for initial symptomatology and negative life events, as well as for the covariates of depressive symptoms. The present findings are consistent with previous research on optimism among adolescents (Chang \& Sanna, 2003; Lai, 2009), and lend support to the prediction that students reporting high level of optimism would be less likely to complain about somatic symptoms, compared to those reporting low levels of optimism. This finding is of interest because few prospective studies among senior high school students have reported significant effects of optimism on later symptomatology. A possible explanation may be that optimism influences physical wellbeing by fostering adaptive behaviours and cognitive responses that are associated with greater flexibility, problem-solving capacity and a more realistic appraisal of negative information.

Notwithstanding, the most interesting finding from the present study was the significant interaction effect of optimism on the relationship between negative life events and somatic symptoms. This finding suggests that an optimistic outlook may be beneficial in buffering the negative effects of stressful life events on somatic symptomatology among the students who are exposed to stressful circumstances in their life. One possible 
explanation for the buffer effect of optimism may be related to the expectations of this optimism in coping with negative life events. When faced with stressful life events, optimists may be likely to believe that positive outcomes are attainable and also to have high expectations of themselves in coping with stressful events, and invent greater efforts to achieve their goals. Unlike pessimistic individuals who tend to use emotion-like coping strategies, optimistic individuals are likely to employ more adaptive coping strategies, such as active and problem-focused coping (Scheier \& Carver, 1985; Mäkikangas \& Kinnunen, 2003).

The presence of protective effects of optimism on somatic symptoms provides support for promoting an optimistic view in adolescents. Recognising the buffering effects of optimism on adolescents could encourage professionals to make more use of this internal psychological resource to alleviate the adverse impact of stressful events on physical health. The teaching of coping skills that can improve adolescent's abilities to handle life demands more effectively may reduce reported somatic symptoms. This may involve adolescents in setting their own goals and developing strategies for overcoming difficulties in their life.

Apart from optimism, previous studies have shown that personality attributes like self-esteem (Mäkikangas \& Kinnunen, 2003), as well as different sources of social support (Murberg \& Bru, 2009; Brisette et al., 2002), moderate the negative impact of stressful events on health among adolescents. Further studies addressing other potential stress moderators together with optimism could provide a better understanding on the role that these factors play in alleviating the negative effects of stressful events on adolescent health.

As stressful life events can lead to increased health costs, higher rates of absence from school and lower performance among the adolescents, it is appropriate to design school-based programmes for those who report high levels of negative life events, as well as for students in general. Such programmes should promote optimistic thinking and seek to modify the school and life environment of stressed students in order to reduce disturbing life experiences. There needs to be both preventative and interventionist dimensions in these programmes. That way, teachers, school psychologists and other professionals will be able to promote a healthy school climate in which stressors are moderated, while at the same time intervening directly when acute problems arise.

Turning to methodological considerations, some things have to be taken into account. A limitation of the present study is that all measures were gathered from participants using self-report questionnaires. This may result in over or underreporting of negative life events and somatic symptomatology. On the other hand, self-report measures may be the most appropriate instruments for the hypotheses considered in this study. Furthermore, as this study uses a convenience sample, it remains to be seen whether the findings can be generalised to more representative sample of adolescent's. Additional prospective studies should seek to use random and diverse sample to make generalisation possible, and also include more objective measures such as recorded sick days and actual usage of health services in the assessment of the individual's health. In addition, studies using lager samples than mine should be undertaken.

A strength of this study compared to most previous studies of the effect of optimism on adolescents health, is the use of a prospective design that make it possible to infer the likelihood of causal relationships between the study variables. However, it should be noted that despite the significant advantages that are associated with using a longitudinal design, findings from the present study do not in themselves provide conclusive evidence of causal linkages between optimism and later somatic symptoms (see Pedhazur, 1982). The use of experimental designs would be appropriate in investigating such potential connections.

\section{REFERENCES}

Arbuckle, J. L., \& Wothke, W. (1999). AMOS 4.0 user's guide. Chicago: SPSS.

Aro, H. (1987). Life stress and psychosomatic symptoms among 14 to 16 years old Finnish adolescents. Psychological Medicine, 17, 191201. doi:10.1017/S0033291700013088

Aromaa, M., Sillanpaa, M., Rautava, P., \& Helenius, H. (2000). Pain experience of children with headache and their families: A controlled study. Pediatrics, 106, 270-275. doi:10.1542/peds.106.2.270

Aspinwall, L. G., \& Taylor, S. E. (1992). Modelling cognitive adaption: A longitudinal investigation of the impact of individual differences and coping on college adjustment and performance. Journal of Personality and Social Psychology, 63, 989-1003. doi:10.1037/0022-3514.63.6.989

Boey, C. C. M., \& Goh, K.-L. (2001). The significance of life-events as contributing factors in childhood recurrent abdominal pain in urban community in Malaysia. Journal of Psychosomatic Research, 51, 559-562. doi:10.1016/S0022-3999(01)00232-X

Brisette, I., Scheier, M. F., \& Carver, C. S. (2002). The role of optimism in social network development, coping, and psychological adjustment during a life transition. Journal of Personality and Social Psychology, 82, 102-111. doi:10.1037/0022-3514.82.1.102

Campo, J. V., \& Fritsch, S. L. (1994). Somatization in children and adolescents. Journal of American Academy of Child and Adolescent Psychiatry, 33, 1223-1235. doi:10.1097/00004583-199411000-00003

Chang, E. C. (1998b). Does dispositional optimism moderate the relation between perceived stress and psychological well-being?: A preliminary investigation. Personality and Individual Differences, 25, 1109-1120. doi:10.1016/S0191-8869(98)00028-2

Chang, E. C., \& Sanna, L. J. (2003). Experience of life hassles and psychological adjustment among adolescents: Does it make a difference if one is optimistic or pessimistic? Personality and Individual Differences, 34, 867-879. doi:10.1016/S0191-8869(02)00077-6

Derogatis, L. R., Lipman, R. S., Rickels, K., Uhlenhuth, E. H., \& Covi, L. (1974). The Hopkins Symptom Checklist (HSCL). A measure of primary symptom dimensions. Modern Problems of Pharmacopsychiatry, 7, 79-110.

Dhossche, D., Ferdinand, R., van der Ende, J., \& Verhulst, F. (2001). Outcome of self-reported functional somatic symptoms in a community sample of adolescents. Annals of Clinical Psychiatry, 13, 191199. doi:10.3109/10401230109147383

Di Lorenzo, C., Coletti, R. B., Lehmann, H. P., Boyle, J. T., Gerson, W. T., Hyams, J. S., Squires, R. H. Jr., Walker, L. S., \& Kanda, P. T. (2005). Chronic abdominal pain in children: A clinical report of the American academy of pediatrics and the North American society for pediatric gastroenterology, hepatology and nutrition. Journal of Pediatric Gastroenterology and Nutrition, 40, 245-248. doi:10.1097/01.MPG.0000155367.44628.21

Egger, H. L., Costello, E. J., Erkanli, A., \& Angold, A. (1999). Somatic complaints and psychopathology in children and adolescents: Stomach aches, musculoskeletal pains, and headaches. Journal of the American Academy of Child and Adolescent Psychiatry, 38, 852-860. doi:10.1097/00004583-199907000-00015

Garber, J., Walker, L., \& Zeman, L. (1991). Somatization symptoms in a community sample of children and adolescents: Further validation of the children's somatization inventory. Psychological Assessment: A Journal of Consulting and Clinical Psychology, 3, 588-593. 


\section{T. A. MURBERG}

Haug, T. T., Mykletun, A., \& Dahl, A. A. (2004). The association between anxiety, depression, and somatic symptoms in a large population: The HUNT-II study. Psychosomatic Medicine, 66, 845-851. doi:10.1097/01.psy.0000145823.85658.0c

Lai, J. C. L. (2009). Dispositional optimism buffers the impact of daily hassles on mental health in Chinese adolescents. Personality and Individual Differences, 47, 247-249. doi:10.1016/j.paid.2009.03.007

Lazarus, R., \& Folkman, S. (1984). Stress, appraisal, and coping. New York: Springer.

Mäkikangas, A., \& Kinnunen, U. (2003). Psychosocial work stressors and well-being: Self esteem and optimism as moderators in a oneyear longitudinal sample. Personality and Individual Differences, 35, 537-557. doi:10.1016/S0191-8869(02)00217-9

Mosing, M. A., Pedersen, N. L., Martin, N. G., \& Wright, M. J. (2010). Sex differences in the genetic architecture of optimism and health and their intercorrelation: A study of Australian and Swedish twins. Twin Research and Human Genetics, 13, 322-329. doi:10.1375/twin.13.4.322

Mulvaney, S., Lambert, E. W., Garber, J., \& Walker, L. S. (2006). Trajectories of symptoms and impairment for pediatric patients with functional abdominal pain: A 5-year longitudinal study. Journal of the American Academy of Child \& Adolescent Psychiatry, 45, 737744. doi:10.1097/10.chi.0000214192.57993.06

Murberg, T. A., \& Bru, E. (2009). The relationships between negative life events, perceived support in the school environment and depressive symptoms among Norwegian senior high school students: A prospective study. Social Psychology of Education, 12, 361-370. doi:10.1007/s11218-008-9083-x

Norusis, M. J. (2008). SPSS 16.0 guide to data analysis. Englewood Cliffs, NJ: Prentice Hall.

Pedhazur, E. J. (1982). Multiple regressions in behavioral research (2nd ed.). New York: Holt, Rinehart \& Winston.

Ryan-Wenger, N. M. (1994). Pychogenic pain in children. Annual Review of Nursing Research, 12, 3-31.

Scheier, M. F., \& Carver, C. S. (1985). Optimism, coping and health: Assessment and implications of generalised outcome expectancies. Health Psychology, 4, 219-247. doi:10.1037/0278-6133.4.3.219

Scheier, M. F., \& Carver, C. S. (1992). Effects of optimism on psychological and physical well-being: Theoretical overview and empirical update. Cognitive Therapy and Research, 16, 201-228. doi:10.1007/BF01173489

Scheier, M. F., Carver, C. S., \& Bridges, M. W. (1994). Optimism, pessimism, and psychological well-being. In E. C. Chang (Ed.), Optimism and pessimism: Implications for theory, research, and practice (pp. 189-216). Washington DC: American Psychological Association.

Skodol, A. E. (1998). Personality and coping as stress-attenuating or amplifying factors. In B. P. Dohrenwend (Ed.), Adversity, stress, and psychopathology (pp. 187-203). New York: Oxford University Press.

Swearingen, E. M., \& Cohen, L. H. (1985b). Measurement of adolescents' life events: The junior high life experiences survey. American Journal of Community Psychology, 16, 204-211.

Taylor, S. E., Kemeny, M. E., Bower, J. E., Gruenewald, T. L., \& Reed, G. M. (2000). Psychlogical resources, positive illusions, and health. American Psychologist, 55, 99-109. doi:10.1037/0003-066X.55.1.99

Ursin, H., Endresen, I. M., \& Ursin, G. (1988). Psychological factors and self-reports of muscle pain. European Journal of Applied Physiology and Occupational Physiology, 57, 282-290. doi:10.1007/BF00635986

Waaktaar, T., Borge, A. I. H., Fundingsrud, H. P., Christie, H. J., \& Torgersen, S. (2004). The role of stressful life events in the development of depressive symptoms in adolescence: A longitudinal community study. Journal of Adolescence, 27, 153-163. doi:10.1016/j.adolescence.2003.09.006

Wagner, B. M., \& Compas, B. E. (1990). Gender, instrumentality, and expressivities: Moderators of the relation between stress and psychological symptoms during adolescence. American Journal of Community Psychology, 18, 383-406. doi:10.1007/BF00938114

Walker, L. S., Garber, J., \& Greene, J. W. (1994). Somatic complaints in pediatric patients: A prospective study of the role of negative life events, child social academic competence, and parental somatic symptoms. Journal of Consulting \& Clinical Psychology, 62, 12131221. doi:10.1037/0022-006X.62.6.1213

Winokur, M., Winokur, D., Rickels, K., \& Cox, D. S. (1984). Symptoms of emotional distress in family planning services: Stability over four-week period. British Journal of Psychiatry, 144, 395-399. doi:10.1192/bjp.144.4.395

Ystgaard, M. (1997). Life stress, social support and psychological distress in late adolescence. Social Psychiatry and Psychiatric Epidemiology, 32, 277-283. doi:10.1007/BF00789040

Ystgaard, M., Tambs, K., \& Dalgard, O. S. (1999). Life stress, social support and psychological distress in late adolescence: A longitudinal study. Social Psychiatry \& Psychiatric Epidemiology, 34, 12. doi:10.1007/s001270050106 